\title{
The Adherence of Musculocutaneous Nerve to Median Nerve Without Piercing Coracobrachialis Muscle - A Case Report
}

\author{
Dawit Habte Woldeyes ${ }^{1}$ \\ 'Department of Anatomy, College of Medicine and Health sciences, Bahir Dar University, po.box 79.
}

\section{Abstract}

The brachial plexus has been reported to show different variations with its formation, course, branches and distribution patterns. During routine dissection in the department of Anatomy, College of Medicine and Health Sciences, Bahir Dar University. One such variation was observed, musculocutaneous nerve arises as usual from the lateral cord but unusually, it does not pierce the coracobrachialis muscle rather, the nerve unites with median nerve and forms common trunk and gives branches to the anterior compartment of the arm in the middle of the arm. Considering clinical importance, recognition and knowledge of such possible anatomical variation will be helpful in the field of neurology, anesthesia and surgery.

Keywords: Brachial plexus, Common trunk, Musculocutaneous nerve, Median nerve, Variation.

Corresponding Author: Dawit Habte Woldeyes, Department of Anatomy, College of Medicine and Health sciences, Bahir Dar University, po.box 79.

Email: dwthabte@gmail.com

Received: August 2018

Accepted: October 2018

\section{Introduction}

Various textbooks, studies and reports clearly demonstrated so far that, the brachial plexus is a complex structure which may be accompanied with variations in the formation of roots, trunks, divisions, cords and terminal branches. ${ }^{[1,2]}$

The musculocutaneous nerve arises from lateral cord at the lower border of pectoralis minor. It leaves the axilla after piercing the coracobrachialis and passes downward between biceps brachii and brachialis muscles in arm. At the junction of middle third and lower third of arm, it appears at the lateral margin of biceps brachii tendon. Here it pierces the deep fascia and passes downward along the lateral aspect of the forearm as the lateral cutaneous nerve of forearm. ${ }^{[3]}$ The musculocutaneous nerve is described as having frequent variations like it may run behind coracobrachialis or pass behind biceps after adhering for some distance to the median nerve. Sometime few fibers of the median nerve may pass to the musculocutaneous nerve, and the median nerve sends a branch to the musculocutaneous nerve. ${ }^{[4]}$ Any variation in course of musculocutaneous nerve in upper arm region can be associated with innervation pattern of coracobrachialis. ${ }^{[5]}$ Evidences reported variations of the musculocutaneous nerve and its branches. It may be absent, ${ }^{[6,7]}$ does not pierce coracobrachialis, ${ }^{[6-8]}$ adheres to the median nerve and passes behind the biceps or obtains branch from median nerve. ${ }^{[9,10]}$
The perforating fibres of musculocutaneous nerve through coracobrachialis muscle makes this nerve to be affected by entrapment neuropathy causing disturbances in skin sensations on the radial part of the forearm and a weakened flexion in the elbow.

The coracobrachialis is an elongated muscle in the superomedial part of the arm. It is a useful landmark for locating other structures in the arm; i.e., the musculocutaneous nerve pierces it, and the distal part of its attachment indicates the location of the nutrient foramen of the humerus. ${ }^{[11]}$ Coracobrachialis muscle is known to have three origins in lower animals, ${ }^{[12]}$ and it is stated that in human the upper two heads fuse and the musculocutaneous nerve passes between them, the third head can occasionally be seen as ligament of Struthers in human. ${ }^{[13]}$ Musculocutaneous nerve is a branch from lateral cord of brachial plexus and during its course it perforates the fibres of Coracobrachialis muscle. ${ }^{[5]}$ There has been a report of the muscle pierced by two nerves. ${ }^{[14]}$

Overall, knowledge on the aforementioned anatomical variation has clinical significance to surgeons, radiologists etc. who interpret plain and computerized imaging. Therefore, the aim of this study is to demonstrate anatomical variation on the existence of musculoutaneous nerve. 


\section{Case Report}

During gross Anatomy dissection session for second year medical students held at Department of Human Anatomy, College of Medicine and Health Sciences; Bahir Dar University, an Anatomical variation was observed in a male adult cadaver. The clinical history of the cadaver is unknown. The pictures from the cadaver were employed and described as follows:

The axillary region of the cadaver was dissected and brachial plexus was examined using standard Anatomy atlas i.e. Cunningham's Manual of Practical Anatomy. ${ }^{[15]}$ On the right side the formation of brachial plexus was normal from roots, trunks, divisions, cord and branches. However, variation was detected on the terminal branch of lateral cord of the plexus, named as musculocutaneous nerve which arises as usual from the lateral cord but unusually it does not pierce the coracobrachialis muscle rather, the nerve unites with median nerve and forms common trunk [Figure 1].

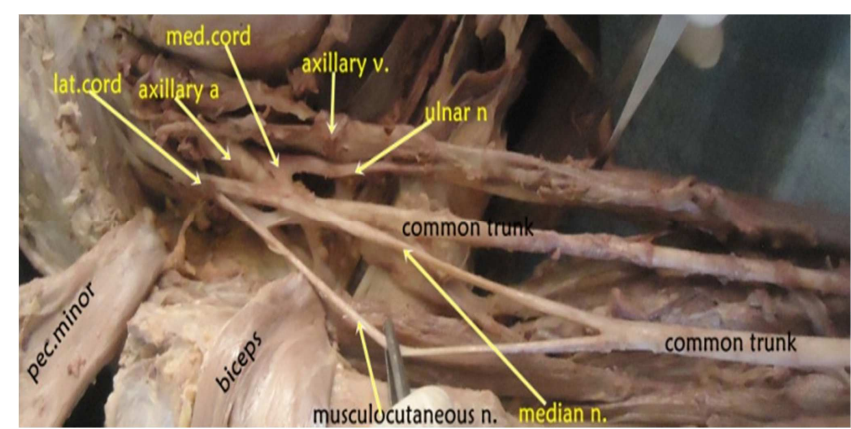

Figure 1: A cadaveric photograph showing the origin of the musculocutaneous nerve and as it joins with the median nerve without piercing the coracobrachialis muscle.

The common trunk which is formed by the union of the two nerves in turn gives rise to several branches to muscles in the middle of anterior compartment of the arm [Figure 2].

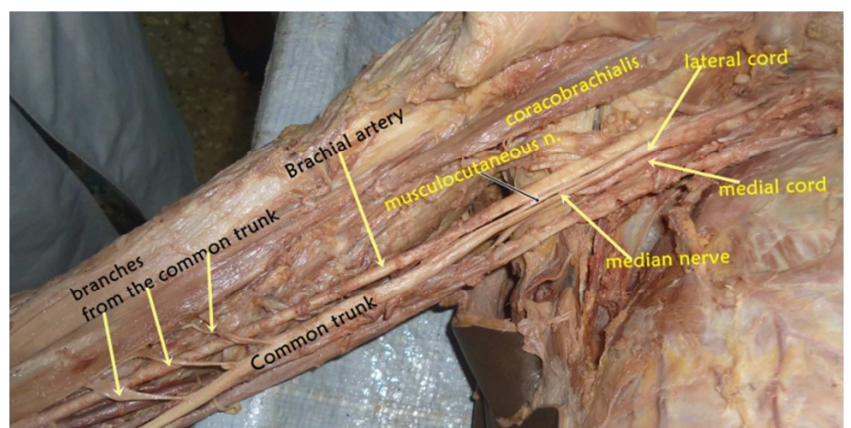

Figure 2: A cadaveric photograph showing the nerve branches originating from the common trunk formed by the union of the musculocutaneous nerve and the median nerve.

\section{Discussion}

Variations in the formation and branching pattern of the brachial plexus constitute an important Anatomical and clinical entity and have been reported by several investigators. Significant variations in nerve patterns may be the result of altered signaling between mesenchymal and neuronal growth cones, ${ }^{[16]}$ or circulatory factors at the time of fusion of brachial plexus cords. ${ }^{[17]}$

Knowledge of the existence of the musculocutaneous nerve median nerve communication in the arm is clinically important; it allows an adequate evaluation and management of upper limb motor disorders caused by peripheral nerve injuries as well as a correct surgical planning and approaches of axilla and arm.

Various authors reported communicating branches between the musculocutaneous and median nerves at different levels, a study done by Loukas and Aqueelah, ${ }^{[18]}$ classified the communication patterns as follows: Type I (45\%): the communications were proximal to the point of entry of the musculocutaneous nerve into the coracobrachialis muscle. Type II $(35 \%)$ : the communications were distal to the point of entry of the musculocutaneous nerve into the coracobrachialis muscle. Type III (9\%): the musculocutaneous nerve did not pierce the coracobrachialis muscle. Type IV (8\%): the communications were proximal to the point of entry of the musculocutaneous nerve and the coracobrachialis, and an additional communication took place distally.

Our report fits well with the character of Type III of the report of the above classification which is the rarest type; it is in line with the studies conducted in India, ${ }^{[9]}$ accordingly, in about $6 \%$ of the cases, the musculocutaneous nerve did not pierce the coracobrachialis muscle instead in the middle one third of the arm, it communicates with median nerve.

In a study to describe the Anatomic variations of the musculocutaneous nerve on 50 upper limbs, it was found that the median nerve was found to not pierce the coracobrachialis in $6 \%$ of the limbs which is a very rare situation; in one case, a common branch arose from the median nerve, just before the insertion of the coracobrachialis, the muscular branches to the muscles of the anterior compartment of the arm arose from that common branch. ${ }^{[19]}$ This finding is similar to the current report but in the present case the nerve does not give branch and does not pierce it and it gives branches to the muscles of anterior compartment around the distal end of the arm.

Variation related to the formation of brachial plexus and the muscles supplied by the branches may be explained in the light of embryogenic development.

The first indication of limb musculature is observed as a condensation of mesenchyme near the base of the limb buds. With further elongation of the limb buds, the muscle tissue splits into flexor and extensor compartments. The upper limb buds lie opposite the lower five cervical and upper two thoracic segments. As soon as the buds form, ventral primary rami from the spinal nerves penetrate into the mesenchyme. At first, each ventral ramus divides into dorsal and ventral branches, but soon these branches unite to form named peripheral nerves which supply extensor and flexor group of muscles respectively. Immediately after this rearrangement of nerves, they enter the limb buds and establish an intimate contact with the differentiating mesodermal condensations and this early contact between the nerve and muscle cells is a prerequisite for their complete functional differentiation. ${ }^{[20]}$ At the infraclavicular level, the lateral fascicle of the brachial plexus usually 
bifurcates giving origin to the musculocutaneous nerve and the lateral root of the median nerve. However, during the embryological development process it is possible that bundles of fibers corresponding to the median nerve initially run together with bundles of fibers of the musculocutaneous nerve. The median nerve recovers the fibers required to perform its motor and sensorial functions in the upper extremity, only when the bundles of fibers were connected with their nerve of origin median nerve at the proximal or mid-thirds of the arm. Although with low frequency, fibers of the musculocutaneous nerve have also been seen to initially run along of the median nerve and later reestablish their configuration through a communicating branch. ${ }^{[21]}$

\section{Conclusion}

These variations have clinical importance in post-traumatic evaluations and exploratory interventions of the arm for peripheral repair. Knowledge of the embryology of neurovascular, muscular and other structures of the body provides valuable information on the existence of multiple variations. The anatomical variations have surgical and/or diagnostic procedure importance.

\section{References}

1. Loukas M, Tubbs RS, Stewart D. An abnormal variation of the brachial plexus with potential clinical significance. West Indian Med. J. 2008; 57: 403.

2. Elizabeth OJ, Marios V, Theano D, Panayotis NS. Neuroanatomy of the brachial plexus: normal and variant anatomy of its formation. Surg. Radiol. Anat. 2010; 32: 291-7.

3. Krishnendu Bhowmik, Arpita Sarkar. Clinically significant unilateral variation of musculocutaneous nerve. Int J Cur Res. 2014; 06 (06):75-79.

4. Standring S. Gray's Anatomy, the Anatomic basis of clinical practice.40th ed. Elsevier: Churchill Livingstone; 2008.

5. Sethi M, Patil S J, Ghosh S. Variant innervation of coracobrachialis muscle: morphological significance, embryological basis and clinical implication. Int $\mathrm{j}$ clin surg adv 2013; 1(2):28-33

6. Jamuna M, Amudha G. A cadaveric study on the Anatomic variations of the musculocutaneous nerve in the infraclavicular part of the brachial plexus. Journal of Clinical and Diagnostic
Research. 2011; 5: 1144-1147.

7. Ajayi N, Lazarus L, Satyapal K. Multiple Variations of the Branches of the Brachial Plexus with Bilateral Connections between Ulnar and Radial Nerves. Int. J. Morphol. 2012; 30:656660 .

8. Pavan P, Anjali G, Rajasekhar H. Variation in the formation of cords of brachial plexus and its relation with axillary artery: Case report. Current Neurobiology. 2012; 3:7-9.

9. Krishnendu B, Arpita S. Clinically significant unilateral variation of Musculocutaneous nerve. International Journal of Current Research 2014; 06:75-79.

10. Vollala R, Potu K, Gorantla R, Reddy S. Variant median nerve and lateral antebrachial cutaneous nerve associated with anomalous brachial vein: case report. Neuroanatomy 2008; 7: 2832 .

11. El-Naggar MM. A study on the morphology of the coracobrachialis muscle and its relationship with the musculocutaneous nerve. Folia Morphol (Warsz) 2001; 60:21724.

12. Rui Diogo and Bernard A. Wood. Comparative anatomy and phylogeny of primate muscles and human evolition. 1st ed. CRC press; 2012.

13. Struthers J. On some points in the abnormal anatomy of the arm. Br for Med Chir Rev 1854; 14:224 -36.

14. Woldeyes DH, Abegaz BA. Coracobrachialis muscle pierced by two nerves: case report. an international journal of experimental and clinical anatomy. $2016 ; 10(2): 148-152$.

15. Romanes GJ. Cunningham's manual of practical Anatomy. Volume 1: $15^{\text {th }}$ ed upper and lower limbs. Oxford: Oxford University Press; 2000.

16. A. Krishnamurthy, SR Nayak, L. Venkatraya Prabhu, RP Hegde, S. Surendran, M. Kumar, MM Pai. The branching pattern and communications of the musculocutaneous nerve. The Journal of Hand Surgery. 2007; 32 (5): 560-562.

17. Sanes HD, Reh TA, Harris WA. Development of the nervous system. 1st ed. New York, Academic Press; 2000.

18. Loukas M, Aqueelah H. Musculocutaneous and median nerve connections within, proximal and distal to the coracobrachialis muscle. Folia Morphol (Warsz) 2005; 64:101-8.

19. Jamuna m., Amudha G. A Cadaveric study on the Anatomic variations of the musculocutaneous nerve in the infraclavicular part of the brachial plexus. Journal of Clinical and Diagnostic Research 2011; 5(6): 1144-1147.

20. T.W. Sadler. Langman's Medical Embryology. 13th ed. Wolters Kluwer; 2015.

21. Luis Ernesto Ballesteros, Pedro Luis Forero, Edna Rocío Buitrago. Communication between the musculocutaneous and median nerves in the arm: an anatomical study and clinical implications. Rev bras ortop. 2015; 50(5):567-572.

Copyright: (C) the author(s), publisher. Academia Anatomica International is an Official Publication of "Society for Health Care \& Research Development". It is an open-access article distributed under the terms of the Creative Commons Attribution Non-Commercial License, which permits unrestricted non-commercial use, distribution, and reproduction in any medium, provided the original work is properly cited.

How to cite this article: Woldeyes DH. The Adherence of Musculocutaneous Nerve to Median Nerve Without Piercing Coracobrachialis Muscle - A Case Report. Acad. Anat. Int. 2018;4(2):4-6.

DOI: dx.doi.org/10.21276/aanat.2018.4.2.2

Source of Support: Nil, Conflict of Interest: None declared. 